\section{Mercury Hydride Ions in the Mass Spectrometer}

Mercury hydride has been studied in optical spectroscopy; but little has been published on the formation of hydrides in the mass spectrometer.

The mass spectrometer used for investigating this field is the sector type ${ }^{1}, 60^{\circ}$ single focusing with 6 -in. radius of curvature, made by the General Electric Company. The pressures were measured on a $V G 1 A$ ion gauge at the tube exit. 'The gauge was calibrated with argon so the hydrogen pressures were actually higher, and in the ionizing region higher by a factor of 10 or more, than the ion gauge pressure recorded.

To study this effect for mercury-hydrogen mixtures, the two gases were held in a sample-system manifold with a leak leading to the spectrometer. Liquid mercury in the manifold at $30^{\circ} \mathrm{C}$. furnished saturated mercury vapour at $2 \cdot 8$ microns pressure. Hydrogen, purified by diffusion through hot palladium, was then added, at varying partial pressures.

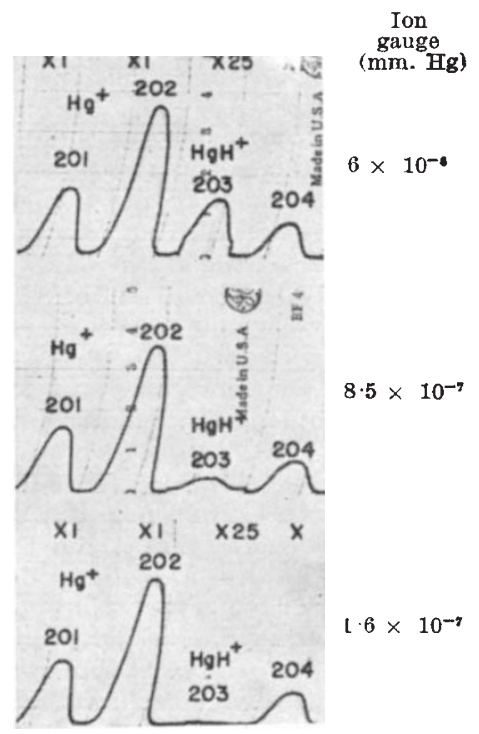

Fig. 1. Mercury hydride ion in excess hydrogen
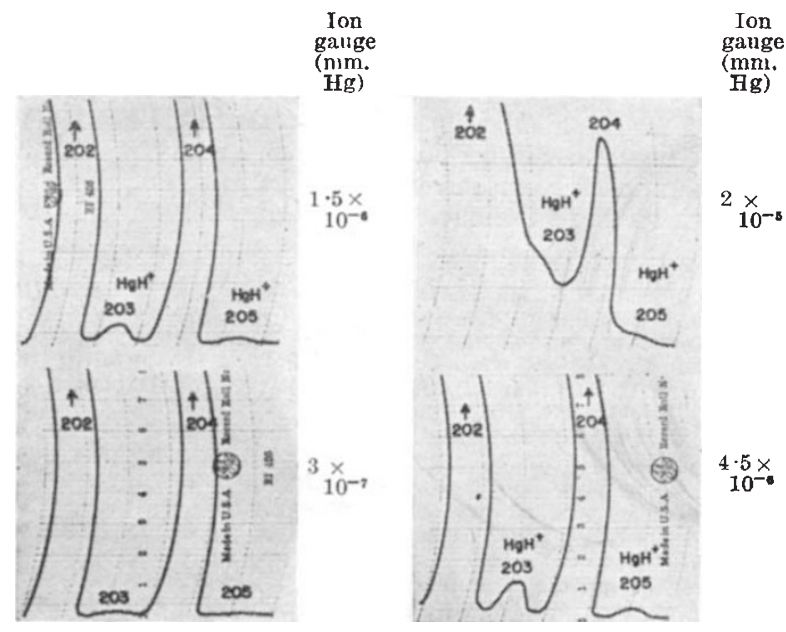

Fig. 2. Loss of resolution at high hydrogen pressure
Spectra were recorded using magnetic scan at $1,500 \mathrm{~V}$. accelerating, $70 \mathrm{~V}$. ionizing, potential.

The hydride ions, ${ }^{2}{ }^{2} \mathrm{HgH}^{+}$and ${ }^{2}{ }^{4} \mathrm{HgH}+$, are shown as peaks in Figs. 1 and 2. The conditions for the mass spectra of Fig. 1 are given in the accompanying table.

\begin{tabular}{|c|c|c|}
\hline $\begin{array}{l}\mathrm{H}_{2} \text { (microns on leak, } \\
\text { McLeod gauge) }\end{array}$ & $\begin{array}{c}\text { Ion gauge } \\
\text { pressure (mm.) }\end{array}$ & $\begin{array}{c}{ }^{202} \mathrm{HgH}+ \\
\text { Peak } 203 \text { intensity } \\
\text { (relative to }{ }^{202} \mathrm{YIg}=100 \text { ) }\end{array}$ \\
\hline $\begin{array}{c}358 \\
101 \\
40 \\
11 \\
0.01\end{array}$ & $\begin{array}{l}6.0 \times 10^{-1} \\
2.0 \times 10^{-6} \\
8.5 \times 10^{-3} \\
4.0 \times 10^{-7} \\
1.6 \times 10^{-7}\end{array}$ & $\begin{array}{c}0.155 \\
0.078 \\
0.039 \\
0.022 \\
\text { less than } 0.002\end{array}$ \\
\hline
\end{tabular}

The intensity of peak $203,{ }^{2} 2 \mathrm{HgH}+$, is proportional to the square root of the hydrogen pressure. This should follow for the reaction : $2 \mathrm{Hg}+\mathrm{H}_{2}=2 \mathrm{HgH}$, where the mercury partial pressure is held constant, and the hydrogen pressure is varied.

In Fig. 2, very high hydrogen pressures with the mercury lead to initial loss of resolution as shown in the series of spectra with decreasing hydrogen pressure. These, all at the same amplification, show ${ }^{204} \mathrm{HgH}^{+}$as well as ${ }^{2}{ }^{2} \mathrm{HgH} \mathrm{H}^{+}$.

Research Laboratory,

Francis J. Norton

General Electric Company, Schenectady, N.Y. Oct. 2.

${ }^{1}$ Nier, A. O., Rev. Sci. Instr., 11, 212 (1940)

\section{Production of the Joshi Effect under $\beta$ - and $\gamma$-Rays}

Examination of the literature having shown absence of information on the Joshi effect under radioactive emanations, a detailed investigation was made employing, in the first instance, a $\beta$-ray capsule with negligible $\gamma$-ray activity. Siemens ozonizer type tubes charged with various vapours and gases and excited by commercial-frequency potentials of kilovolts were used. In agreement with results obtained with visible ${ }^{1}$ and ultra-violet $^{2}$ radiation and with X-rays ${ }^{3-6}$, and employing especially organic vapours', an exposure to $\beta$-rays caused an instantaneous and reversible positive effect $+\Delta i$ at relatively low voltage; with slight increase of voltage, $+\Delta i$ diminished sharply; and finally changed over to $-\Delta i$ abuve $V_{m}$, the threshold for a 'self-maintained' discharge. Thus, for example, cyclohexane vapour showed under $\beta$-rays about 150 per cent positive Joshi effect at $4 \mathrm{kV}$.; +9.5 per cent and $-5 \cdot 8$ per cent at 6 and $8 \mathrm{kV}$. respectively.

Simultaneously with the production of $+\Delta i$, highfrequency pulses appeared in the current structure, lasting only during exposure to $\beta$-rays, when observed with a cathode ray oscillograph. $-\Delta i$ obtained at higher voltages corresponded to an instantaneous and reversible decrease of the amplitude of the high-frequency pulses. In an electronegative gas, only $-\Delta i$ was detected: it decreased with the overvoltage $V-V_{m}$; thus, for example, with chlorine at $110 \mathrm{~mm}$. mercury pressure, $-\Delta i$ was 11.4 and $2 \cdot 4$ per cent at $4 \mathrm{kV}$, and $20 \mathrm{kV}$. respectively.

The action of a Geiger-Müller counter can be usefully compared with the mechanism of an ozonizer 